\title{
Handbuch sexualisierte Gewalt - aus Fehlern lernen
}

\section{Dr. med. Werner Tschan}

Ehemaliger Programmleiter, CAS Intervention und Prävention bei sexualisierter Gewalt, Universität Zürich

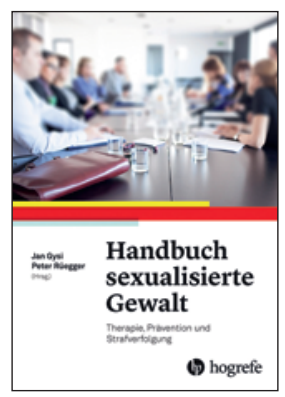

hogrefe Im Umgang mit Sexualdelikten spielen Unwissenheit und fehlende fachliche Kompetenz eine zentrale Rolle. Schon alleine die Tatsache, dass das FBI die von 1929 stammende Definition über «Vergewaltigung» 2012 revidieren musste, verdeutlicht, wie sich selbst ausgewiesene Fachleute bei der Theamtik auf einem Minenfeld bewegen - offenbar war man jahrzehntelang von falschen Voraussetzungen ausgegangen. Das Fachbuch von Gysi und Rüegger (Hrsg.) bringt die Erfahrung und die Sichtweise von 62 Fachleuten aus unterschiedlichen Disziplinen zusammen - Ziel der Herausgeber ist die Optimierung der Helferkette - von Opferberatungsstellen über Medizin, Psychotherapie, Forensik, Polizei, Justiz und Ethik - vermittelt durch einen transdiziplinären Dialog der involvierten Fachleute. Dieser Diskurs muss auf Augenhöhe geführt werden - wo beispielsweise die Justiz einfordert, dass sich die Akteure den Anforderungen der Strafprozesse anzupassen haben, da muss umgekehrt gelten, dass die Justiz die Opferanliegen resp. das Aussageverhalten bei sexualisierter Gewalt gebührend berücksichtigt. Dabei prallen unterschiedliche Perspektiven aufeinander, welche zu zahlreichen Konflikt- und Dilemmasituationen führen. Die Herausgeber haben es verstanden, durch die vielen unterschiedlichen Sichtweisen das Wissen - inkl. den bestehenden Lücken und Defizten - umfassend darzustellen.

Den beteiligten Autor(inn)en gelingt es, die prekäre Situation der Opfer von Sexualdelikten im heutigen System sehr anschaulich darzustellen - alle Fachdisziplinien bemühen sich redlich um eine optimale Aufarbeitung; aber erst im transdisziplinären Dialog zeigt sich die bedrückende Sachlage. Stets liegt die Beweislast auf Seiten der Betroffenen. Das Buch ist deshalb sehr geeignet, für die Konzeption von disziplinenübergreifenden Aus-, Fort-, und Weiterbildungen zur Thematik hilfreiche Anregungen zu vermitteln.
Die heutigen Sichtweisen und Kenntnisse über Täterstrategien haben die individualpathologische Sichtweise abgelöst - wie einzelne Beiträge aufzeigen, mit erheblichen Konsequenzen für die Rechtssprechung wie auch für die Behandlung.

Leider ist es den Herausgebern, abgesehen von vereinzelten Hinweisen (z.B. S. 243), nicht gelungen, die bisherige Genderperspektive in Bezug auf sexualisierte $\mathrm{Ge}$ walt zu überwinden. Der "conformity bias» - dass man in der Forschung das bestätigt, wovon man überzeugt ist - spielt gerade bei dieser Thematik und deren praktischen Umsetzung eine zentrale Rolle. Sexualisierte Gewalt ist eine Menschenrechtsverletzung und kein Genderproblem. Dies illustriert beispielsweise die eingangs erwähnte Neufomulierung der US-amerikanischen Bundespolizei FBI. Frauen wie Männer sind gemäss den heutigen Erkenntnissen etwa gleich häufig durch sexualisierte Gewaltdelikte betroffen; Frauen als Täterinnen werden weitaus häufiger erkannt, als bisher von vielen Fachleuten angenommen wird. Es muss deshalb vertieft reflektiert werden, wie sexualisierte Gewalt verstanden wird, welche strafrechtlichen Normen dem jeweiligen Verständnis zu Grunde liegen, und wie die Forschung diese Definitionen aufgreift. Das Handbuch ist durch die Kooperation der beiden Herausgeber - Gysi als Arzt/Psychiater und Rüegger als Jurist/Opferanwalt - eine umfassende Darstellung des aktuellen Kenntnisstandes im Bereich der Sexualdelinquenz und deren Aufarbeitung gelungen. Das Werk verdeutlicht prägnant, wie sich in der Rechtssprechung eine parteiliche Haltung gegenüber den Opfern etablieren muss - hat die Justiz doch klar die Verfassungsgrundsätze umzusetzen, welche den Schutz der persönlichen und sexuellen Integrität postulieren. $\mathrm{Zu}$ wenig werden aus juristischer Sicht neuere Tendenzen berücksichtigt - wie beispielsweise Beiträge von «therapeutic jurisprudence» oder der Täter-Opfer-Ausgleich (restorative justice) als Teil des Straf- und Massnahmevollzugs.

Neuland betreten die Herausgeber in der Berücksichtigung ethischer Anforderungen an die Opferberatung, die Rechtssprechung, die forensische Medizin und den Strafund Massnahmevollzug im Bereich der sexualisierten Gewalt. Das Buch liefert damit grundlegende Denkanstösse und Lösungsansätze in Umgang mit der Thematik. 